\title{
Noise Radar with Microwave Correlation Receiver
}

\author{
W. SuseK*, B. Stec And Cz. RećKo \\ Institute of Radioelectronics, Faculty of Electronics, Military University of Technology \\ S. Kaliskiego 2, 00-908 Warsaw, Poland
}

\begin{abstract}
The idea of microwave quadrature correlation as well as the block diagram of the microwave broadband correlation receiver and principle of quadrature correlation detection of noise signals using an analog microwave correlator are presented in the paper. Measurement results for the correlation function of noise signals are shown and application of such solution in the noise radar for precise determination of distance changes and velocity of these changes is also presented. Results for short range noise radar operation are presented both for static and moving. Experimental results using 2.6-3.6 GHz noise like waveform for the signal from a breathing human is presented. Conclusions and future plans for the use of presented detection technique in broad-band noise radars close the paper.
\end{abstract}

PACS: 07.57.Hm, 41.90.+e, 84.40.Ua, 84.40.Xb, 84.40.Dc

\section{Introduction}

Noise radars belong to radars which use both noise or pseudo-noise waveforms as probing signals and correlation techniques for noise signals [1]. As far as correlation reception is concerned, either a delay line of constant or variable parameters is necessary in the receiver system, or spectral interferometric techniques, executed with digital methods in programmable digital systems, should be used. Noise radars, despite of the appearances, can be successfully applied in measurements of the target range, target velocity as well as in terrain or target imaging (SAR, ISAR) [2]. Noise radars can be also used for search of objects hidden behind screens for infrared radiation. An example of a noise radar using analog correlation technique in a microwave band is presented in the paper.

\section{Direct correlation detection of the noise signal}

Correlation-type receiver is a typical element, among others, entering into the noise radar. Their basic parameters are: broad band of a signal, low power density and capability to obtaining high sensitivity of receiving devices using non-conventional methods [3]. In particular, the principle of correlation coherent detection of the noise signal allows operation of many devices in the same frequency range without mutual interference. The idea of the direct correlation detection of the noise signal transmitted and received by the noise radar is presented in this section $[4,5]$. The analysis of detector operation was carried out on the basis of schematic diagram shown in Fig. 1. Let us assume that the transmitter generates a signal in the form of a narrowband noise of Gaussian distribution with mean value equal to zero and the variance equal to $\sigma^{2}$. Signals in the individual points of the system can be described in time domain as follows:

\footnotetext{
* corresponding author; e-mail: Waldemar.Susek@wat.edu.pl
}

$$
S_{\mathrm{N}}(t)=X(t) \cos \left(\omega_{0} t\right)-Y(t) \sin \left(\omega_{0} t\right),
$$
where $X(t)$ and $Y(t)$ are the independent stationary random processes of Gaussian distribution with mean values equal to zero and $\omega_{0}$ is the median angular frequency of the band occupied by noise signal

$$
\begin{aligned}
& S_{\mathrm{NDL}}(t)=k_{1} S_{\mathrm{N}}\left(t-T_{\mathrm{DL}}\right), \\
& S_{\mathrm{O}}(t)=k_{2} S_{\mathrm{N}}\left(t-\frac{2 D}{c}\right),
\end{aligned}
$$

where $D$ is the instantaneous distance between the radar and the object, $T_{\mathrm{DL}}$ is the time delay in the delay line, $\mathrm{c}$ is the speed of light and $k_{1}, k_{2}$ are the signal attenuation coefficients for delay line and propagation space, respectively. Relation describing the instantaneous distance has the form

$$
D=\frac{c T_{0}}{2}+v t+D_{r} \cos \left(\omega_{r} t\right)
$$

In relation (4) $T_{0}$ is the signal delay time on the radar-object-radar path, $v$ is the radial velocity of the object, while $D_{r}$ and $\omega_{r}$ are the amplitude and the pulsation of the additional harmonic movement of certain object parts, respectively. The object as a whole can move with radial velocity $v$ or stay in rest. In result of multiplication of $S_{\mathrm{NDL}}(t)$ and $S_{\mathrm{O}}(t)$ as well as $S_{\mathrm{OP} / 2}(t)$ and $S_{\mathrm{NDL}}(t)$, and next low-pass filtration (integration) with time constant $T_{\mathrm{p}}$, two expressions for quadrature output signals $I(\tau, t)$ and $Q(\tau, t)$ can be found in the form of

$$
\begin{aligned}
& I(\tau, t) \\
& \quad=A(\tau) \cos \left(\omega_{0} \tau+\frac{2 \omega_{0} v t}{c}+\frac{2 \omega_{0} D_{r}}{c} \cos \left(\omega_{r} t\right)\right), \\
& Q(\tau, t) \\
& \quad=A(\tau) \sin \left(\omega_{0} \tau+\frac{2 \omega_{0} v t}{c}+\frac{2 \omega_{0} D_{r}}{c} \cos \left(\omega_{r} t\right)\right),
\end{aligned}
$$

where $\tau=T_{0}-T_{\mathrm{DL}}$.

Relations (5) and (6) describe quadrature components of the correlation function of the noise with limited band. A complex representation of the correlation function $R(\tau, t)$ of the narrowband noise signal can be formed 


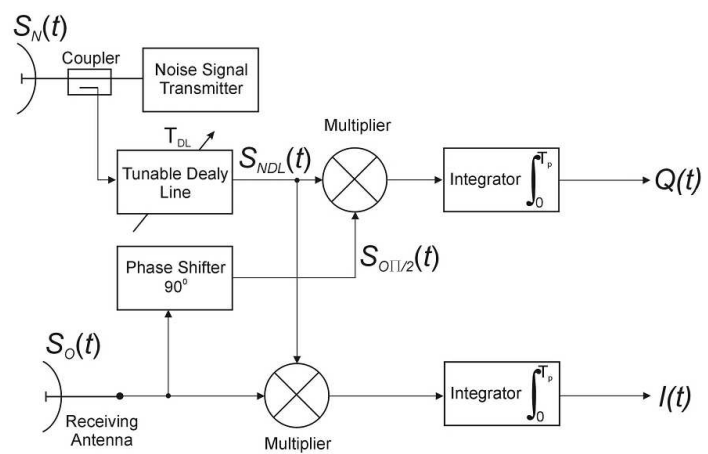

Fig. 1. Schematic diagram of quadrature detection of the noise signal in the correlation detector.

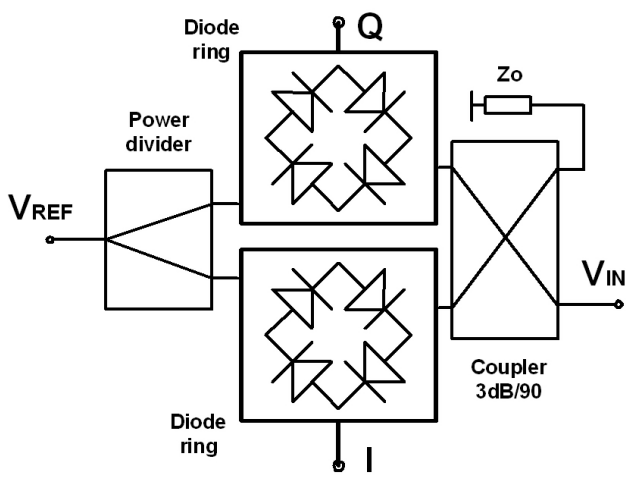

Fig. 2. Microwave quadrature correlator with diode rings.

in this case as

$$
R(\tau, t)=I(\tau, t)+\mathrm{j} Q(\tau, t) .
$$

It results from the presented analysis that in the equipment built according to the schematic diagram presented in Fig. 2, in contrary to digital processing, the correlation function of the narrowband noise signal is determined. If $R(\tau, t)$ is the complex representation of the correlation function of the narrowband noise signal, then the instantaneous amplitude $A$, phase $\varphi$ and pulsation $\omega$, similarly to analytical signal, amount to, respectively

$$
\begin{aligned}
& A(\tau)=\frac{k_{1} k_{2}}{T_{\mathrm{p}}} \int_{0}^{T_{\mathrm{p}}}\left[X\left(t-T_{0}\right) X\left(t-T_{\mathrm{DL}}\right)\right. \\
& \left.\quad+Y\left(t-T_{0}\right) Y\left(t-T_{\mathrm{DL}}\right)\right] \mathrm{d} t \\
& \varphi(t)=\arctan (Q(t) / I(t)) \\
& \omega(t)=\frac{\mathrm{d}}{\mathrm{d} t} \varphi(t) u .
\end{aligned}
$$

The relation (10) is of particular meaning in the presented mode of noise signal detection. It shows that it is possible to detect insignificant movements of the object with respect to the observational noise radar. The more dynamic changes of the instantaneous phase the better precision of the object movement detection.

\section{Microwave quadrature correlator}

The scheme of signals multiplier is presented in Fig. 3. This circuit presents the typical layout of double balanced mixer. Assuming that the signals at the inputs of such a circuit are given by

$$
U_{1}=U_{m 1} \cos \left(\omega t+\varphi_{1}\right), \quad U_{2}=U_{m 2} \cos \left(\omega t+\varphi_{2}\right),
$$

where $\varphi_{1}$ - phase of signal $U_{1}$ and $\varphi_{2}$ - phase of signal $U_{2}$, then the current signals across the diodes can be expressed as

$$
\begin{aligned}
& i_{\mathrm{D} 1}=a_{0}+0.5 a_{1}\left(-U_{1}-U_{2}\right)+0.5 a_{2}\left(-U_{1}-U_{2}\right)^{2}, \\
& i_{\mathrm{D} 2}=a_{0}+0.5 a_{1}\left(-U_{1}+U_{2}\right)+0.5 a_{2}\left(-U_{1}+U_{2}\right)^{2}, \\
& i_{\mathrm{D} 3}=a_{0}+0.5 a_{1}\left(U_{1}+U_{2}\right)+0.5 a_{2}\left(U_{1}+U_{2}\right)^{2}, \\
& i_{\mathrm{D} 4}=a_{0}+0.5 a_{1}\left(U_{1}-U_{2}\right)+0.5 a_{2}\left(U_{1}-U_{2}\right)^{2} .
\end{aligned}
$$

By adding and subtracting appropriate currents it can be proved that constant values are compensated and the output current can be expressed as

$$
i_{\text {out }}=i_{\mathrm{D} 1}-i_{\mathrm{D} 2}+i_{\mathrm{D} 3}-i_{\mathrm{D} 4}=2 a_{2} U_{1} U_{2} .
$$

Introducing (11) into (13) the final relation describing the output current can be written as

$$
\begin{aligned}
& i_{\text {out }}=a_{2} U_{m 1} U_{m 2} \cos (2 \omega t+\Delta \varphi) \\
& +a_{2} U_{m 1} U_{m 2} \cos (\Delta \varphi) .
\end{aligned}
$$

A low-pass filter at the output of signals multiplier makes it possible to consider only the low frequency component and on that condition the output current. The voltage at the output of low-pass filter is given by

$$
U_{\text {out }}=k U_{m 1} U_{m 2} \cos (\Delta \varphi),
$$

where $k$ - factor of proportionality, $U_{m 1}, U_{m 2}$ - signal amplitudes and $\Delta \varphi=\varphi_{1}-\varphi_{2}$ - phase difference. The basic element of diode ring is a Schottky barrier diode.

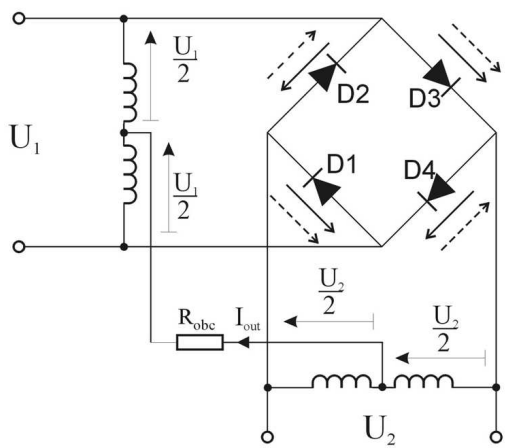

Fig. 3. The equivalent circuit of correlator with diode ring.

The broadband microwave quadrature correlator is a modified six-port measurement module. The system consists of one power divider, one coupler, and two diode rings. Input voltages $V_{\mathrm{REF}}$ and $V_{\mathrm{IN}}$ are divided into two equal parts and delivered to diode rings with adequate phase shifts. Diode rings play a role of multiplication systems. 


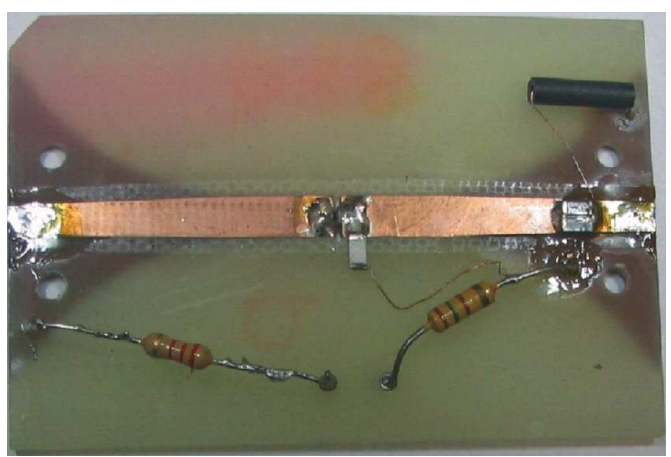

Fig. 4. View of the upper side of the planar structure of the microwave multiplication system with transformers.

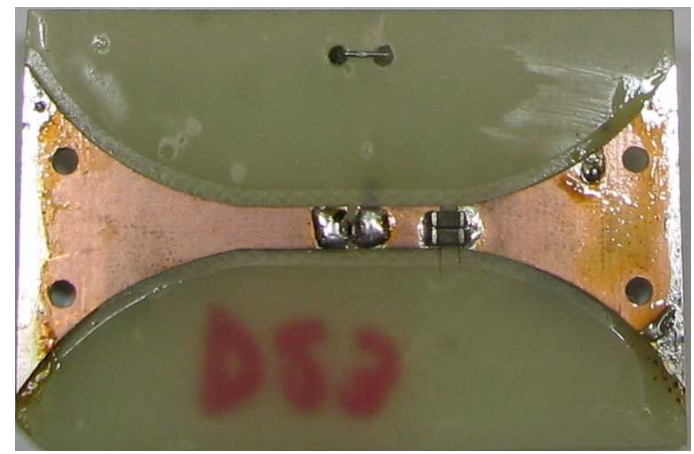

Fig. 5. View of the bottom side of the planar structure of the microwave multiplication system with transformers.

The diode ring is connected to double conductor line which changes over to asymmetric strip line at the inputs of the $V_{\text {REF }}$ and $V_{\text {IN }}$ signals. Such transitions, in the microwave technique, realize functions of transformers, shown in Figs. 4, 5. The suggested detector is a broadband microwave quadrature correlator. Detailed analysis of the system shown in Fig. 2 is presented in Refs. [5, 6].

\section{Broadband microwave correlation-type receiver for noise signals}

The broadband microwave correlation-type receiver consists of input circuits, low-noise microwave amplifier, broadband microwave quadrature correlator, low-frequency amplifiers with high gain, and the system for information display. Input circuits were equipped with interchangeable microwave filters made with microstrip lines technique. The filters band could be changed from $100 \mathrm{MHz}$ to $1 \mathrm{GHz}$, and their median frequency equaled $3 \mathrm{GHz}$. The low-noise amplifier is a broadband system with $40 \mathrm{~dB}$ gain and the noise factor not worse than $1.5 \mathrm{~dB}$. At the quadrature detector output two signals exist (signals $I$ and $Q$ described by relations (5) and (6), respectively). These signals are gained in measurement amplifiers with adjustable voltage gain from 100 up to 10000.
The band of the correlator output signals is limited in low-pass filters. The frequency range of the post-detection band changes from parts of $\mathrm{Hz}$ up to $100 \mathrm{~Hz}$. Signals prepared in the above described way are then digitized in the $\mathrm{A} / \mathrm{D}$ converter and next sent to $\mathrm{PC}$ via USB link. The PC plays a role of the processing and information display system. The noise signal transmitter is the mean power noise generator. The microwave noise generator was constructed on the basis of semiconductor original noise source and a sequence of amplifiers and microwave filters. Therefore a noise signal source was achieved, operating at the median frequency $f_{0}=3 \mathrm{GHz}$ with $3 \mathrm{~dB}$ band $B=700 \mathrm{MHz}$ and the spectral power density $G_{\mathrm{N}}=-78 \mathrm{dBm} / \mathrm{Hz}$. The spectrum of noise power was finally formed via output band-pass filter of a uniformly flat transmittance profile.

\section{Measurement results}

For random courses, contrary to expectation, instantaneous values of these runs do not change in an arbitrary way, since there exists a certain relation between them resulting, among others, from the slow response real electronic circuits. For estimation of this relation an autocorrelation function $R\left(t_{1}, t_{2}\right)$ of the process $X(t)$ is used which is the associative moment of the random variables $X\left(t_{1}\right)$ and $X\left(t_{2}\right)$. The normalized autocorrelation function of white noise at the output of the ideally rectangular band-pass filter with the band $B=f_{2}-f_{1}$ and the median frequency $f_{0}=\left(f_{2}+f_{1}\right) / 2$ is illustrated in Fig. 6 and described by dependence (16)

$$
\frac{R(\tau)}{R(0)}=\left[\sin \left(2 \pi f_{2} \tau\right)-\sin \left(2 \pi f_{1} \tau\right)\right] / 2 \pi \tau B,
$$

where $\tau=t_{2}-t_{1}$ is the delay time.

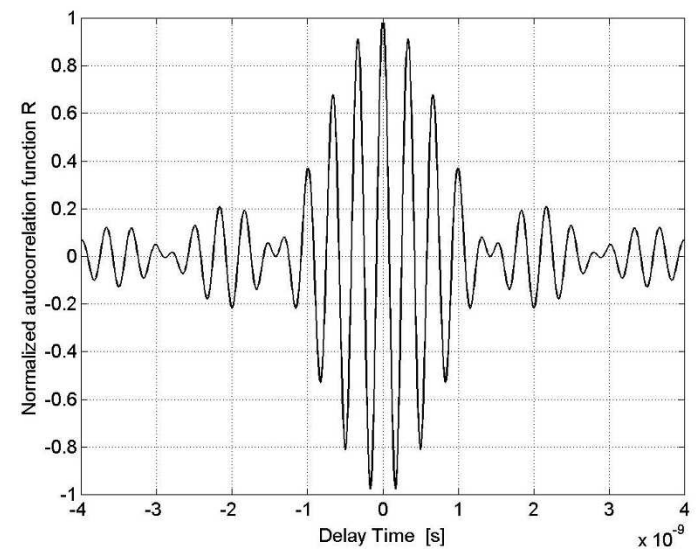

Fig. 6. Normalized autocorrelation function of white noise at the output of an ideally rectangular band-pass filter.

A distance to the object and the object radial velocity were measured in the system presented in Fig. 7. The noise radar constructed according to the block diagram presented in Fig. 3 was used for this purpose. A horn re- 


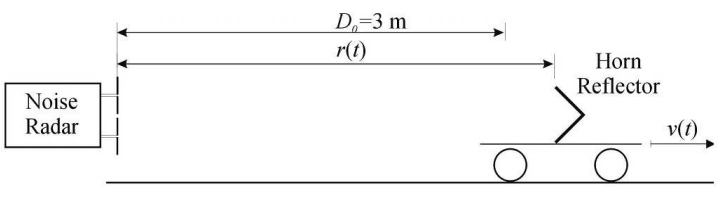

Fig. 7. Measurement setup diagram.

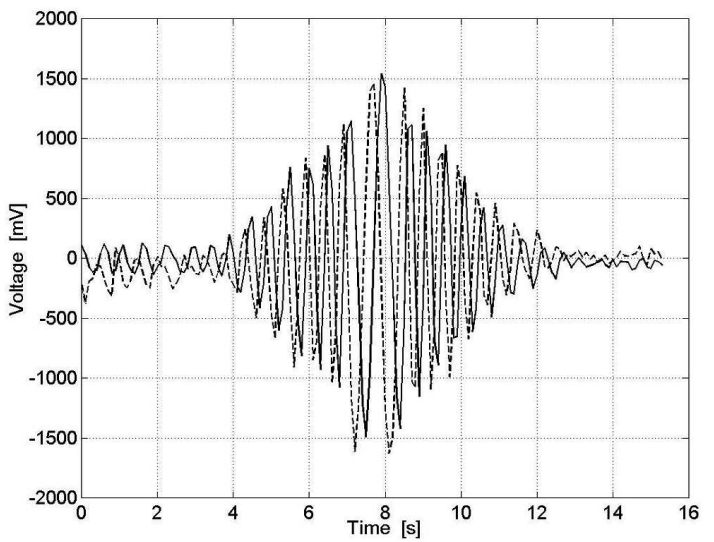

Fig. 8. Courses of the voltage changes at the outputs $I$ (solid line) and $Q$ (dotted line) of the microwave quadrature correlator.

flector moving on the dielectric carriage with average velocity of the $v=0.1 \mathrm{~m} / \mathrm{s}$ was applied as the measured object. The adjustable delay line of the noise radar was set to the constant delay value corresponding to the radarobject distance equal to $D_{0}=3 \mathrm{~m}$. Measurement result of the correlation function of narrowband noise signals in form of two courses $I$ and $Q$ obtained at the output of the microwave quadrature correlator is presented in Fig. 8. The maximum of the registered courses appears at the distance $r=D_{0}=3 \mathrm{~m}$ which corresponds to delay time $\tau=0$. The change of delay time $\tau$ was obtained via change of the horn reflector distance $r(t)$ from the noise radar according to the dependence

$$
\tau=\left(r-D_{0}\right) / c .
$$

For very high frequencies in the microwave range the analog system of the correlative detector can be realized since for these frequencies digital realization of the autocorrelation function is not available. Using courses $I$ and $Q$ registered on the output of the microwave correlator, a Doppler frequency was calculated in the specified time range in which the object was moving with variable velocity. For the case under discussion the pulsation described by the relation (10) is the Doppler pulsation. So, the Doppler frequency can be calculated from the following dependence:

$$
f_{\mathrm{D}}(t)=\omega(t) / 2 \pi \text {. }
$$

The result of the Doppler frequency calculation is presented in Fig. 9. It results from the courses $I$ and $Q$ shown in Fig. 10 that for the analyzed time movement velocity of the object changed. The Doppler frequency changed in the parallel way. It reaches the maximum value of $-2.4 \mathrm{~Hz}$ for the time $6.8 \mathrm{~s}$ which corresponds to the velocity $v(6.8)=0.12 \mathrm{~m} / \mathrm{s}$, and the minimum value of $-0.8 \mathrm{~Hz}$ for the time $7.15 \mathrm{~s}$ which corresponds to the velocity $v(7.15)=0.04 \mathrm{~m} / \mathrm{s}$. The noise radar with microwave correlating receiver can measure instantaneous radial velocity, which is changing in time domain. But the measurement of variable radial velocity is difficult for the interpretation reasons in the frequency domain.

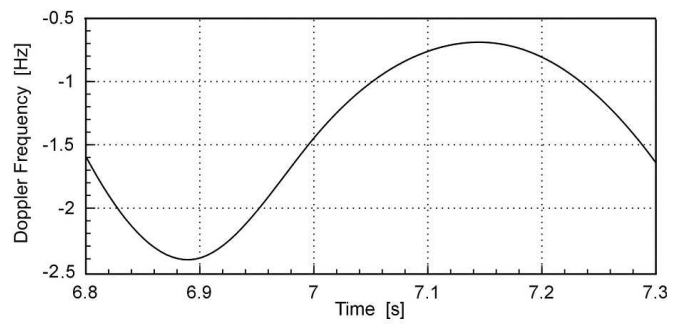

Fig. 9. Doppler frequency courses during the object movement.

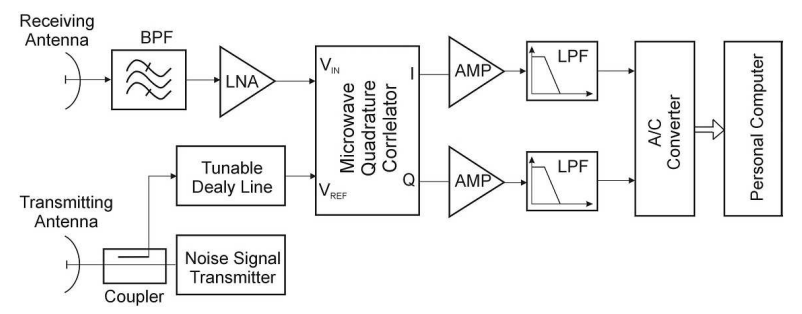

Fig. 10. Block diagram of the noise radar with microwave correlation-type receiver.

Next example for the noise radar with microwave correlator using is living organism vital functions observation, such as breathing. The lung volume increasing and decreasing phenomenon is used for this. The lung volume changing causes the chest movement. This movement is detected by noise radar successfully. This movement can be modelled as harmonic wave, whose amplitude corresponds to the chest maximum raising and the frequency corresponds to the move frequency of inspiration and expiration. In Eqs. (5) and (6), the $\left(2 \omega_{0} D_{r} / c\right) \cos \left(\omega_{r} t\right)$ component is the mathematical description of the assumed breathing model. The measurement results are presented in Fig. 11. The measurement was made for the setup presented in Fig. 12. The sitting on the chair man with the regular breathing was used as the object for the tests. The correlator output voltages $I$ and $Q$ can be described as the modified Eqs. (5) and (6) in the way

$$
\begin{aligned}
& I=A(0) \cos \left(\frac{2 \omega_{0}}{c} D_{r} \cos \left(\omega_{r} t\right)\right), \\
& Q=A(0) \sin \left(\frac{2 \omega_{0}}{c} D_{r} \cos \left(\omega_{r} t\right)\right) .
\end{aligned}
$$

Equations (5) and (6) modification corresponds with the fact that the tested man was placed in the distance 
$D_{0}=3 \mathrm{~m}$. This value gives as the maximum correlation function for the transmitted and received by the radar noise signal. Additionally, the man as the object was motionless (radial velocity $v=0$ ). From Fig. 11 it is clear to understand the fact that using analogue microwave correlator circuit, the breathing can be detected, neglecting complicated digital signal processing. Using the correlator circuit, the internal correlation function structure information is not losed. For example, for the center frequency of $3 \mathrm{GHz}$, the wavelength is $10 \mathrm{~cm}$ in the open air, so the distance of the tested object from the radar changing at $2.5 \mathrm{~cm}$, gives the output correlator voltages changing in the full range from the minimum to the maximum values. This fact was used for the living organism activity detection.

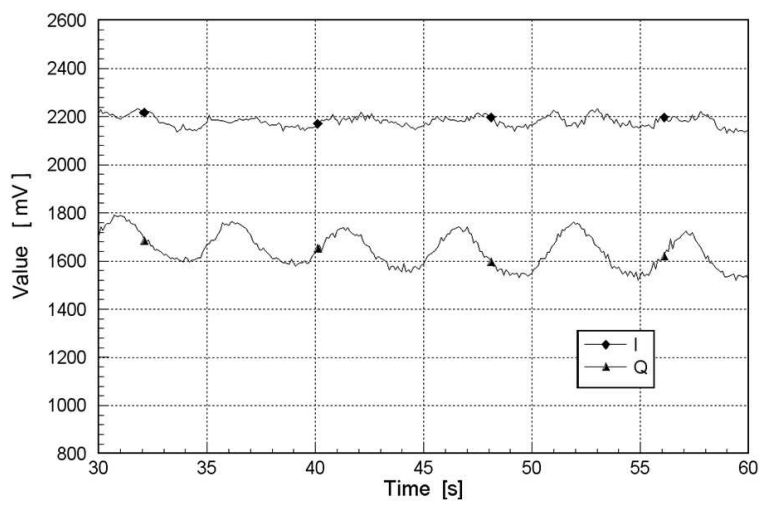

Fig. 11. Courses of the voltage changes at the outputs $I$ and $Q$ of the microwave quadrature correlator for the signal from a breathing human.

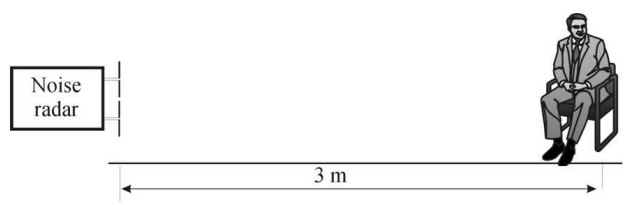

Fig. 12. Setup diagram for breathing human measurement.

\section{Summary}

The suggested correlation detection method of broadband noise signals allows construction of radars with noise signals. These radars can be used, among others, for monitoring living beings activities displaying, for instance, movements of individual parts of their bodies. For very high frequencies in the microwave range ana$\log$ systems of the correlative detector can be realized since for these frequencies digital realization of the autocorrelation function is not available. Noise radars are characterized by a "pin-type" undetermination function. Therefore, a distance and Doppler frequency can be precisely and unambiguously determined by means of these radars. Noise signals coming from other sources operating at the same frequency range will not be correlated with the specific signal of the noise radar, thus the values of correlation functions of these signals will be very low. There appears a possibility of construction of anti-collision radars for moving objects which will not jam each other even for huge number of devices in specified area. Frequency range for noise radars covers an interval from $\mathrm{MHz}$ to hundreds of $\mathrm{GHz}$ and this is only a technological limitation at the current stage of electronics development. Main application fields of noise radars are as follows: anti-collision radars [8], protection of objects, detection of motion/movement [9], recognition and penetration of inaccessible objects, penetration of objects hidden in shallow soil layers, or detection of living beings in inaccessible areas. The authors' future plans will focus on application of the presented in the paper detector systems for selected noise radars.

\section{Acknowledgments}

This work was supported by the Ministry of Science and Higher Education for the years 2010-2012 under Development Project No. O R00 006211.

\section{References}

[1] S.R.J. Axelsson, IEEE Trans. Geosci. Remote Sensing 42, 2370 (2004).

[2] S.R.J. Axelsson, IEEE Trans. Geosci. Remote Sensing 41, 2703 (2003).

[3] Z. Li, R. Narayanan, IEEE Trans. Aerospace Electron. Syst. 42, 904 (2006).

[4] W. Susek, B. Stec, Metrology Measur. Syst. XVII, 289 (2010).

[5] B. Stec, Bull. WAT 423, 71 (1987) (in Polish).

[6] B. Stec, in: Int. Defence Conf., Abu Dhabi 1995.

[7] K.A. Lukin, A.A. Mogyla, Y.A. Alexandrov, O.V. Zemlyaniy, T. Lukina, Yu. Shiyan, in: The Fourth Int. Symp. on Physics and Engineering of Millimeter and Sub-Millimeter Waves, Vol. 2, Kharkov, 2001, p. 870.

[8] C. Lai, R.M. Narayanan, Q. Ruan, A. Davydov, IET Radar Sonar Navig. 2, 244 (2008).

[9] B. Stec, C. Rećko, in: XIII Int. Microwave Conf., Vol. III, Wrocław 2000, p. 65. 